\title{
A School Violence Detection Algorithm Based on a Single MEMS Sensor
}

\author{
Jifu Shi ${ }^{1}$, Liang Ye ${ }^{1,2, *}$, Hany Ferdinando ${ }^{2,3}$, Tapio Seppänen ${ }^{4}$, Esko Alasaarela ${ }^{2}$ \\ 1. School of Electronic and Information Engineering, Harbin Institute of Technology, \\ Harbin, China \\ 2. Optoelectronics and Measurement Techniques Laboratory, Department of Electrical \\ Engineering, University of Oulu, Oulu, Finland \\ 3. Department of Electrical Engineering, Petra Christian University, Surabaya, Indonesia \\ 4. Department of Computer Science and Engineering, University of Oulu, Oulu, Finland \\ shijifu1993@126.com, yeliang@hit.edu.cn, hferdina@ee.oulu.fi, tapio@ee.oulu.fi, \\ esko.alasaarela@ee.oulu.fi
}

\begin{abstract}
School violence has become more and more frequent in today's school life and caused great harm to the social and educational development in many countries. This paper used a MEMS sensor which is fixed on the waist to collect data and performed feature extraction on the acceleration and gyro data of the sensors. Altogether nine kinds of activities were recorded, including six daily-life kinds and three violence kinds. A filter-based Relief-F feature selection algorithm was used and Radial Basis Function (RBF) neural network classifier was applied on them. The results showed that the algorithm could distinguish physical violence movements from daily-life movements with an accuracy of $93 \%$.
\end{abstract}

Keywords: Activity recognition, School violence, Relief-F, MEMS accelerometer, RBF neural network

\section{Introduction}

School violence is a worldwide problem, and relevant survey data showed that $50 \%$ of the students admitted to the existence of bullying behavior, whereas $47 \%$ of the students confirmed that they have been hurt by verbal bullying or physical violence. It has caused many students to fear campus life and seriously affects students' physical and mental health as well as their studies. Throughout recent years, there is a common problem of many campus violence that bullied students are inflicting psychological shadows on their young hearts because they are afraid or threatened not to inform parents or teachers about their being bullied. Traditional campus monitoring has the problem of blind area because of geographical location restriction, and cannot realize active detection so as to prevent violence efficiently.

With the development and popularization of various smart wearable devices, smartphones and bracelets have become the most common wearable sensor devices. These devices have built-in sensors, including optical sensors, inertial sensors (accelerometers and gyroscopes), GPS locators, and so on. Many scholars use these 
technologies to identify human daily behaviors and recognize complex behaviors. In this situation, the study of active detecting of school violence has come into being. We use active sensors which are built into wearable devices on the students to actively detect campus violence in real time and transmit the results to teachers or parents in an active and timely manner. This active detection avoids being bullied and undiscovered, and can solve school violence problems in a timely and effective manner.

The remainder of this paper is organized as follows: In section 2, we review the related work on activity recognition; in section 3, we describe the entire system framework and physical bullying detection algorithm; in section 4, we present the classification results; and the conclusion is given in Section 5.

\section{Activity Recognition}

Study of school bullying was originally initiated by some European scholars for passive methods. Along with the popularity of smartphones on campus, there have been a number of school bullying detection schemes which are based on the intelligent mobile phone. For example, "Stop Bullies": there is a shortcut key on the mobile phone, we can press this key to send photos of the incident location and geographical location to the intended recipients when suffered bullying. However, a common drawback of these detection schemes is passive detection, the victims are often too late to operate or dare not to operate when they are suffering from bullying, which greatly reduces the detection rate and does not solve school bullying in a timely and effective manner.

With the development of pattern recognition technology, great progress has been made in the field of physical activity recognition. The physical activity recognition system using single or multi-sensors is used in real life. Some scholars have used the accelerometer to obtain good results in many fields. Reference [1] used a three-axis accelerometer to propose an accurate physical activity recognition system for medical monitoring and rehabilitation. A patient wore a wireless acceleration sensor to identify six daily movements and transition events. Feature selection was performed using SFFS and the naive Bayes and KNN classification algorithms were adopted. The recognition accuracy rate was 95\%. In addition, PA (Physical Activity) recognition systems based on hand-held mobile terminals have become a research hotspot [2]-[5]. In [6], accelerometers and gyroscopes in smartphones were used to identify daily activities, and the recognition accuracy could reach about $85 \%$. However, considering the battery capacity and memory size of handheld mobile terminals, reducing the complexity of the algorithm has also become the optimization goal for many scholars [7]. Multi-sensor data fusion enables a more comprehensive capture of human movements compared with a single sensor [8]-[12]. This paper proposes a posterior-adapted class-based fusion of multi-accelerometers data algorithm by using waist and leg sensor data. The results show that the recognition accuracy is improved compared with a single sensor. 


\section{Methods}

The system framework includes data collection and preprocessing, feature extraction, normalization, feature selection and classifier design. This study considers time efficiency and resource utilization. Algorithm complexity is priority for the design of feature selection algorithms and classifiers in this paper.

\subsection{Data Acquisition and Pre-processing}

The background of this research project is for the campus violence scene. In order to simulate campus violence scenes more realistically, data acquisition was performed indoors with protective gears and motion sensors. Data acquisition consisted of ten subjects who were asked to perform six types of daily-life activities (jump, play, run, walk, stand, and fall-down) and three bullying activities (beat, push, and push-down). The position of the motion sensor on the waist is shown in Fig. 1.

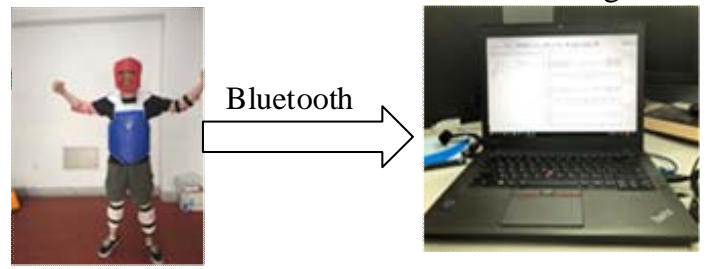

Fig. 1. The process of data acquisition

The sensor sampled data at a sampling rate of $50 \mathrm{~Hz}$ and included tri-axial accelerometer and tri-axial gyroscope signals. In the process of data acquisition, we recorded video to remove non-experimental data to establish a database of bullying. In addition, the raw data was smoothed by using wavelet filtering because it contained random noise and jitter noise. The y-axis of the tri-axial accelerometer and the gyroscope is the vertical direction, and the $\mathrm{x}$-axis and the z-axis are the horizontal directions. For the acceleration, the $\mathrm{x}$-axis and the $\mathrm{z}$-axis are combined in the horizontal direction, i,e.,

$$
A C C_{\text {Hori }}=\left(A C C_{x-a x i s}(i)^{2}+A C C_{z-a x i s}(i)^{2}\right)^{0.5}
$$

For the gyroscope data, it is combined as follows.

$$
\text { Gyro }=\left(\operatorname{Gyro}_{x-a x i s}(i)^{2}+\text { Gyro }_{y-a x i s}(i)^{2}+\operatorname{Gyro}_{z-a x i s}(i)^{2}\right)^{0.5}
$$

After the preprocessing of these data, these signals are segmented into windows with a $50 \%$ overlap between two consecutive windows. In this case, every decision made on the activity is for the duration of the window. 


\subsection{Feature Extraction}

After data pre-processing, a large set of features were extracted from the each window for the $A c c_{y-a x i s}$, $A c C_{\text {Hori }}$, Gyro. First, feature extraction is performed within each window of the data in the time domain, and then FFT is carried out to extract the features in the frequency domain.

Table 1 lists the extracted features from each window. These features were based on previous PA research [14]-[16].

Table 1. List of features extracted

\begin{tabular}{llc}
\hline No & Features & count \\
\hline 1 & Mean & 3 \\
2 & Median difference & 3 \\
3 & Maximum value & 3 \\
4 & Minimum value & 3 \\
5 & Differential maximum & 3 \\
6 & Differential mean & 3 \\
7 & Standard deviation & 3 \\
8 & Kurtosis & 3 \\
9 & Skewness & 3 \\
10 & Cross-correlation of ACC axis & 3 \\
11 & 25 $5^{\text {th }}$ percentile & 3 \\
12 & $75^{\text {th }}$ percentile & 3 \\
13 & Zero-crossing rate of ACC axis & 3 \\
14 & Energy of frequency domain & 3 \\
15 & Mean of frequency domain & 3 \\
16 & Median difference of frequency & 3 \\
17 & Maximum value of frequency & 3 \\
\hline
\end{tabular}

\subsection{Normalization \& Feature Selection}

Data normalization processing is a basic work of data mining. Different evaluation indicators often have different dimensions and dimension units. In order to eliminate the dimensional impact among different indicators, we need to standardize data to solve the data comparability of indicators. Normalization is required to limit the feature in a certain range. The Z-score normalization was applied in this paper. For example, feature $y$ can be normalized by the following formula,

$$
\widehat{y_{i}}=\frac{y_{i}-\bar{y}}{\sigma}
$$

where $\bar{y}$ and $\sigma$ are mean and standard deviation, respectively. 
Feature selection algorithm is one of the most critical steps that affect the recognition accuracy of the entire system. Selecting good features can increase the recognition accuracy, but useless features can cause over-fitting and lower recognition accuracy, and increase algorithm complexity. Therefore, it is necessary to select the best feature subsets for all the extracted features to classify. The filter-based Relief-F feature selection algorithm is applied to select the most useful feature set in this study. This selection algorithm is efficient, simple, and feature selection can be performed without building a classifier. In this article, the data were used to calculate the correlations between feature and each labelled activity, and then a threshold was set to select feature subsets with greater correlation for training and testing.

\subsection{Classification Algorithms}

The design of the classifier needs to consider practical applications and classification performance. Research work shows that the BP neural network has very limited learning speed due to its global approaching nature, and it has great limitations in some cases with high real-time requirements. The Convolutional Neural Network (CNN) based on deep learning has the advantages of no need to manually select features and excellent classification performance, but training requires a large amount of sample data and high performance equipment. Compared with BP and CNN, RBF has the advantages of simple structure, fast convergence, and ability to approximate any nonlinear function. The generalized RBF classifier was adopted based on this project for real-time and data resources. According to the analysis of experimental results (see Section 4 for details), the RBF has a very high classification performance and can accurately distinguish between school bullying activity and daily-life activity.

\section{Classification Performance}

\subsection{Evaluation Approach and Indicators}

Five-fold cross validation is used to validate the classifier performance. This method ensures that all data will undergo training and testing, and it can effectively avoid the occurrence of over-fitting or under-fitting. It can be more persuasive and practical.

The confusion matrix is used to perform a contingency table analysis of the true class and the predicted class. For each instance, the number of true-negatives (TN), false-negatives (FN), false-positives (FP) and true-positives (TP) are calculated by comparing the truth labels and the predictions. The performance of the system can be evaluated by several parameters such as accuracy, precision, recall and F1-score. The formulas for these parameters are calculated as follows, 
accuracy $=\frac{T P+T N}{P+N}$

precision $=\frac{T P}{T P+F P}$

recall $=\frac{T P}{T P+F N}$

The F1-score is used to comprehensively consider the accuracy and recall rate, and it can be calculated by

$$
F_{1} \text {-Score }=\frac{2}{\frac{1}{\text { precision }}+\frac{1}{\text { recall }}}
$$

\subsection{Classification Results}

The activity recognition conversion matrix is shown in Table 2. The first column of the table represents the actual activity. The figures in the table represent the probability that the actual activity is recognized as the activity in the first row, and the diagonal indicates the accuracy of the activity recognition. Finally, statistics are divided into violent activities and non-violence activities. The "push", "push-down”, and "beat" are classified as violent activities, while other activities are classified as non-violent activities. Violent activity recognition confusion matrix is shown in Table 3.

Table 2. Conversion ratio of physical activity recognition (unit: \%)

\begin{tabular}{cccccccccc}
\hline & Beat & Jump & Play & Push & Run & Stand & Walk & $\begin{array}{c}\text { Push- } \\
\text { down }\end{array}$ & $\begin{array}{c}\text { Fall- } \\
\text { down }\end{array}$ \\
\hline Beat & $\mathbf{7 6 . 3}$ & 0.0 & 3.0 & 5.9 & 0.0 & 2.2 & 4.4 & 5.9 & 2.3 \\
Jump & 0.0 & $\mathbf{1 0 0 . 0}$ & 0.0 & 0.0 & 0.0 & 0.0 & 0.0 & 0.0 & 0.0 \\
Play & 1.1 & 0.0 & $\mathbf{9 0 . 0}$ & 3.3 & 0.0 & 0.0 & 5.6 & 0.0 & 0.0 \\
Push & 15.7 & 0.0 & 2.9 & $\mathbf{7 1 . 4}$ & 0.0 & 1.4 & 8.6 & 0.0 & 0.0 \\
Run & 0.0 & 0.0 & 0.0 & 0.0 & $\mathbf{9 9 . 1}$ & 0.0 & 0.0 & 0.9 & 0.0 \\
Stand & 2.0 & 0.0 & 3.3 & 0.0 & 0.0 & $\mathbf{9 4 . 0}$ & 0.7 & 0.0 & 0.0 \\
$\begin{array}{l}\text { Walk } \\
\text { Push- }\end{array}$ & 0.0 & 0.0 & 1.4 & 2.1 & 0.0 & 0.0 & $\mathbf{9 6 . 5}$ & 0.0 & 0.0 \\
down & 15.6 & 0.0 & 4.4 & 8.9 & 0.0 & 4.4 & 0.0 & $\mathbf{5 5 . 6}$ & 11.1 \\
$\begin{array}{c}\text { Fall- } \\
\text { down }\end{array}$ & 12.0 & 0.0 & 2.0 & 6.0 & 0.0 & 6.00 & 4.0 & 26.0 & $\mathbf{4 4 . 0}$ \\
\hline
\end{tabular}


Table 3. Confusion matrix of PA recognition (unit: \%)

\begin{tabular}{ccc}
\hline & Non-violence & Violence \\
\hline Non-violence (actual) & 92.8 & 7.2 \\
Violence (actual) & 16.2 & 83.8 \\
\hline
\end{tabular}

The evaluation index can be calculated from the PA recognition confusion matrix. The recognition accuracy is about $93 \%$ and the recall reaches approximately $83 \%$. The experimental results show that we can distinguish between violent activities and non-violent activities accurately.

\section{Conclusion}

School bullying has a great influence on the physical and mental health of teenagers. This paper proposes an active violence detection algorithm which analyzes the accelerometer and gyro data collected by sensors. After features extraction and selection and classifier design, we can distinguish between violent activities and daily-life activities. Totally nine types of activities were tested, and the average recognition ratio was approximately 93\%. This method can be applied to students' wearable devices with built-in sensors to better protect students and prevent violence in schools.

\section{Acknowledgements}

This work was supported by the National Natural Science Foundation of China (61602127), and partly supported by the Directorate General of Higher Education, Indonesia (2142/E4.4/K/2013), and the Finnish Cultural Foundation, North Ostrobothnia Regional Fund. The authors would like to thank those people who have helped with these experiments.

\section{References}

[1] Gupta P, Dallas T. Feature selection and activity recognition system using a single triaxial accelerometer[J]. IEEE Transactions on Biomedical Engineering, 2014, 61(6):1780.

[2] Politi O, Mporas I, Megalooikonomou V. Human motion detection in daily activity tasks using wearable sensors[C]// Signal Processing Conference. IEEE, 2014:2315-2319.

[3] Altini M, Vullers R, Van Hoof C, et al. Self-calibration of walking speed estimations using smartphone sensors[C]// IEEE International Conference on Pervasive Computing and Communication Workshops. IEEE Computer Society, 2014:10-18. 
[4] Charlene V. San Buenaventura, Tiglao. Basic Human Activity Recognition Based on Sensor Fusion in Smartphones. 2017 IFIP/IEEE Symposium on Integrated Network and Service Management (IM).

[5] Wang Z, Huo Y. A multi-attribute fusion acceleration feature selection algorithm for activity recognition on smart phones[C]// International Conference on Information Science, Electronics and Electrical Engineering. IEEE, 2014:145-148.

[6] Coskun D, Incel O D, Ozgovde A. Phone position/placement detection using accelerometer: Impact on activity recognition[C]// IEEE Tenth International Conference on Intelligent Sensors, Sensor Networks and Information Processing. IEEE, 2015:1-6.

[7] Ayu M A, Ismail S A, Mantoro T, et al. Real-time activity recognition in mobile phones based on its accelerometer data[C]// International Conference on Informatics and Computing. IEEE, 2017:292-297.

[8]Gao L, Bourke A K, Nelson J. A system for activity recognition using multi-sensor fusion[C]// International Conference of the IEEE Engineering in Medicine \& Biology Society, Embc. Conf Proc IEEE Eng Med Biol Soc, 2011:7869.

[9]Kushwah A, Kumar S, Hegde R M. Multi-sensor data fusion methods for indoor activity recognition using temporal evidence theory[J]. Pervasive \& Mobile Computing, 2015, 21:19-29.

[10] Rahman M M, Charoenlarpnopparut C, Suksompong P. Signal processing for multisensor E-nose system: Acquisition and classification[C]// International Conference on Information, Communications and Signal Processing. 2015:1-5.

[11] Gao L, Bourke A K, Nelson J. A comparison of classifiers for activity recognition using multiple accelerometer-based sensors[M]. 2012.

[12] Zebin T, Scully P J, Ozanyan K B. Inertial Sensor Based Modelling of Human Activity Classes: Feature Extraction and Multi-sensor Data Fusion Using Machine Learning Algorithms[M]// eHealth 360 . Springer International Publishing, 2017.

[13] Chowdhury A, Tjondronegoro D, Chandran V, et al. Physical Activity Recognition using Posterior-adapted Class-based Fusion of Multi-Accelerometers data[J]. IEEE Journal of Biomedical \& Health Informatics, 2017, PP(99):1-1.

[14] Gao L, Bourke A K, Nelson J. Evaluation of accelerometer based multi-sensor versus single-sensor activity recognition systems[J]. Medical Engineering \& Physics, 2014, 36(6):779-785.

[15] S. Pirttikangas, K. Fujinami, and T. Nakajima, "Feature selection and activity recognition from wearable sensors," in Ubiquitous Computing Systems, ed: Springer, 2006, pp. 516-527.

[16] Wichit N. Multi-sensor data fusion model for activity detection[C]// International Conference on ICT and Knowledge Engineering. IEEE, 2015:54-59. 